\title{
Intelligent Products: A Spinal Column to Handle Information Exchanges in Supply Chains
}

\author{
Damien Trentesaux ${ }^{1}$, Bernard Grabot ${ }^{2}$, and Yves Sallez ${ }^{1}$ \\ ${ }^{1}$ Université Lille Nord de France, UVHC, Tempo-Lab., F-59313 Valenciennes, France \\ \{Damien.Trentesaux, Yves.Sallez\} @univ-valenciennes.fr \\ ${ }^{2}$ Université de Toulouse, INP/LGP-ENIT, Avenue d'Azereix, \\ BP 1629, F-65016 Tarbes Cedex, France \\ bernard.grabot@enit.fr
}

\begin{abstract}
This paper outlines some practical problems linked to information exchange occurring in nowadays supply chains, with a special emphasis on the aeronautical sector. The Intelligent Product paradigm is presented as an adequate solution to address some of these problems and to provide rapid gains along the whole product life-cycle. The paper intends to illustrate how the IP paradigm could bring natural solutions to historical problems in supply chains, in rupture with the way they are usually addressed. A short case study describes how the suggested concepts could help to address real problems in nowadays supply chains.
\end{abstract}

Keywords: Intelligent product, collaborative network, supply chain, information system.

\section{Introduction}

In today's global market, companies no longer compete as independent entities but as integral part of supply chains (SC) [Min and Zhou, 2002]. Joint-planning so that cooperation and strategic partnerships over the entire SC are now universally considered as conditions for building more efficient and reactive manufacturing networks [Arkan et al., 2012 ; Ding et al., 2011], but sharing information and knowledge is recognized as a pre-requisite for such achievements [Krause et al., 2007]. Nevertheless, answering to the basic questions: "what information to share?" and "how to share it?" remains a difficult task. In this communication, we underline some practical requirements for information sharing, and suggest addressing the second question using the Intelligent Product (IP) paradigm, considered as a natural physical and informational link between partners of the SC.

In section 2 are provided examples of information sharing in collaborative networks. The concept of intelligent products is introduced in section 3, and is applied to the problem of information sharing in SC in section 4. A short case study describes in section 5 how the suggested concepts could help to address real problems in nowadays supply chains. 


\section{Information Exchange in Collaborative Networks}

Focusing on their core business, nowadays companies work in larger and larger networks but the necessity to be more reactive and flexible induces the paradoxical requirement to efficiently share information and knowledge all along the SC: information and knowledge sharing are nowadays considered as keys for improving the performance of manufacturing systems, internally as well as with external partners [Wadwha et al., 2010]. Moreover, information sharing is an essential condition for developing trust between partners, trust being considered as necessary to maintain long term collaborative SC relationship [Nyaga et al., 2010; Ren et al., 2010].

Which information to share is the object of a large literature, but how to share it is also a difficult question, which has motivated many studies in various domains. Industrial Information systems provide a first element of answer, nowadays ERP (Enterprise Resource Planning) or APS (Advanced Planning Systems) allow for instance a company to share production plans with its suppliers. Web portals are another technology allowing to easily provide information, and are more and more used in Supply Chains (see for instance Sup@irWorld, the web portal allowing Airbus to communicate with its suppliers1). At the same time, Excel ${ }^{\mathrm{TM}}$ sheets are still the most common mean to exchange information in Supply Chains...

In most of the cases, information is "pushed" by the large companies, their suppliers (especially the smallest ones) having often difficulties for adequately introducing the provided information in their own information systems. A project called APOSAR has recently been performed in the South-West of France aiming at analysing the relationships between large and small companies involved in aeronautical supply chains [Ming et al., 2013]. Twenty companies were visited in that purpose: seven large ones and thirteen of middle (around 200 employees) or low (less than $100 \mathrm{em}$ ployees) size, working in various technical domains of the aeronautical field. The following seven requirements for improving information exchange in supply chains are one of the results of this study. Information exchange:

- should be possible internally and externally with the same tools (Req. \#1),

- should be secure, i.e. should not require to open an access to the main information system of the company (Req. \#2),

- should be consistent with Concurrent Engineering approaches, i.e. should support communication between the actors of the product lifecycle (Req. \#3),

- should favour trust, and should be automated, so that the partners can focus on the strategic issues of collaboration (Req. \#4),

- should use tools allowing a good consistency with traceability systems, which are often dedicated tools (Req. \#5),

- should be based on tools which use could be extended to the late steps of the product lifecycle (delivery, usage, recovery, reuse/recycling) (Req. \#6),

- should allow an easy access of actors, for instance using mobile devices (Req. \#7).

\footnotetext{
${ }^{1}$ http://www.airbus.com/tools/airbusfor/suppliers/
} 
As shown in next sections, the intelligent product may be a natural vector for coping with these requirements.

\section{Intelligent Products}

Many definitions of Intelligent Products (IP) can be found in the literature, see for example [McFarlane et al., 2002]. In [Meyer et al., 2009] was proposed a typology defining three main types of IP: identified IP are at least able to manage their own information, given by sensors, RFID readers and other techniques; active IP are able to memorize information, communicate and trigger events or notify users when there is a problem (e.g. IP has fallen, its temperature is too high); decisional IP are able to execute decisional algorithms, including possible learning mechanisms. In [Sallez et al., 2010a] was proposed a generic model of active intelligent products based on the concept of augmentation enabling classical passive products to become intelligent and active. A proof-of-concept of active intelligent products in the context of manufacturing has been then implemented (see Figure 1) [Sallez et al., 2010b].

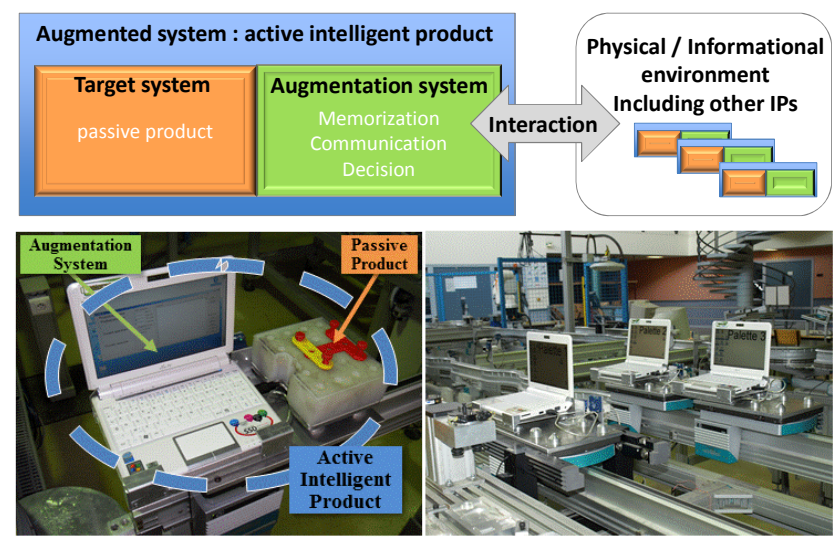

Fig. 1. Active intelligent product in manufacturing: concept and proof-of-concept

In this implementation, the augmentation system was embedded along the passive product, but this is not compulsory: a RFID tag can be used to identify an IP and a remote centralized computer system can support all the augmentation systems. An advantage of remote implementations is related to costs, while drawbacks concern the rise of complexity with the increasing number of managed IPs, the risk to centralize all the information in case of failure, and lastly, to handle information far away from the real physical systems where events occurs. In fact, one of the will to use IP is to bring decisional, informational and communicational capabilities close to the physical world, reducing decision lags and increasing local dynamic solving of disturbances. In a PLM (Product Lifecycle Management) context, the IP can be seen as the vector that crosses all the different phases of the product lifecycle. In that sense, IP is a possible way to implement Closed-loop PLM [Kiritsis, 2011]. In this context, information exchanges between IP and the different crossed information systems allow better 
knowledge capitalization and a better interoperability among systems. The IP is also a natural vector of interoperability since it carries the data along the whole life-cycle, formatted according to a pre-determined and commonly agreed ontology.

\section{IP: A Communication Vector between Customer and Supplier}

In manufacturing, IP have been widely proposed for real time production control inside companies. Few works exploiting the potential benefits of IP have been done at the SC level and most of them use level 1 (identified) IP [Sarac et al., 2010] with typical instantiation of the IP concept using RFID technology. The objective to use level $1 \mathrm{IP}$ in SC is to reduce inventory losses and increase information accuracy. The two other levels of IP (level 2: active and level 3: decisional) are still not really exploited in the context of SC, even if some works exist (see for example [Yang et al., 2009]). The interest to use levels 2 and 3 IP in the context of SC, the "intelligent capabilities" being embedded or not into the products, is pointed out in next sections.

\subsection{IP as the Spinal Column in Supply Chains}

Let us first assume that the IP information system is composed of two distinct parts: a public part, which follows the product when it is sent to the different partners, and a private part, aiming at internal information sharing, cleared when the product leaves a company. Let us also assume that the SC can be modelled as a direct graph where nodes are companies, and vertices are flows of products between two companies. Under these assumptions, the suggested basic principles may be summarized as follows:

- the manufacturing process of the product is firstly analysed and each node of the SC requiring to gather or modify information stored in the IP is identified.

- A technology is chosen and implemented for giving intelligent functionalities to the product (embedded or distant). The granularity (i.e. an augmentation system for $m$ passive products) is also chosen. In the proof-of-concept of Fig. 1, $m$ was set to 1 but this value typically depends on parameters such as production lot size, total value of the product, required cost/constraint for embedding technology, etc.

- The part of the information system concerned with the shared information is attached to the information system of the IP (embedded or distant part of the augmentation system), including triggering or decisional capabilities.

- This local information system is initiated with i) data collected during the successive planning phases performed all along the SC (due date for the focal company, deduced due date for tier 1 suppliers, etc.) ii) constraints of down stream partners to be taken into account by up stream partners.

- The IP information system is periodically synchronized with the main information system using communication terminals (fixed or mobiles).

This behaviour is summarized in Figure 2. Information is first prepared to be attached to the augmentation systems in the planning-based phases (1) and (2). Then the instantiation of the IP (on each branch of the supply chain, only one being represented 
in Fig. 2) is made (3). During manufacturing (4), at each convergent node of the SC (e.g., assembly, light blue and dark blue nodes), a synthesis of the information brought by each component is performed.

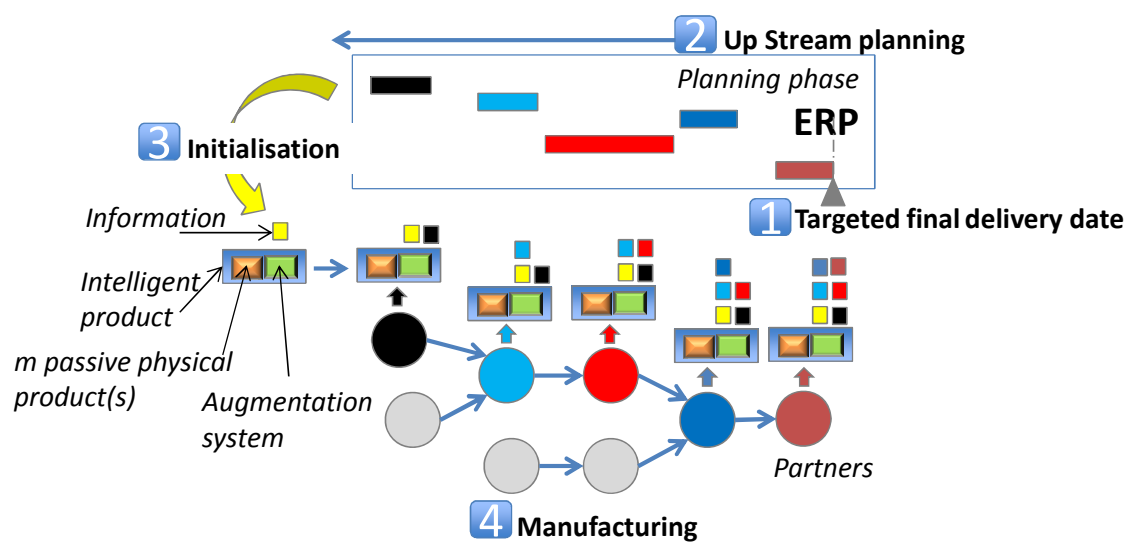

Fig. 2. The intelligent product as a communication vector: basic principle

\subsection{Addressing the Listed Requirements on Information Exchange Using IP}

Using the proposed principles, the IP becomes the interface among the different actors and information systems all along the SC. This section exhibits how the IP paradigm may help to address the requirements listed in section 2 , focusing on information exchange issues.

Answer to req. \#1: From planning to delivery, information gathering and processing is logically organised around the IP. Since the product is the only element crossing the whole SC, using it as a physical support is logical. Moreover, it is easier to interface each system with a common one (the IP) than having to design several 1-1 interface systems for all the stakeholders of the SC.

Answer to req. \#2: The IP limits this problem, since it is possible to only associate to it what is required for the next part of its lifecycle, then to make the information accessible to the downstream partners. There is no need to open an access to an internal database.

Answer to req. \#3: As introduced above, the utilisation done with the carried information may be memorized by the IP itself and reused in the lifecycles of its successors. For instance, a recurrent problem in maintenance can be due to a production quality problem or to a design problem (e.g. undersized part). This allows tightening the links between actors of the SC and facilitates the improvement of successive versions of passive products using this capitalized knowledge gathered by previously used, maintained or dismantled IP.

Answer to req. \#4: The IP is an "objective" (tangible) element, exchanged between customer and supplier. It is therefore a trust carrier in their relationship, via 
unequivocal traceability indicators (e.g. time spent in transportation, average endured, lost time in inventory, condition violation in transportation, etc.).

Answer to req. \#5: In the IP, the information system is associated to the passive product during all the phases of its lifecycle: traceability becomes a natural function.

Answer to req. \#6: If needed and authorized, the collection of information may continue during the usage of the customer, thanks to the multiple networks now available in the private life. According to the closed-loop PLM concept, different forward and backward information flows are supported by the IP.

Answer to req. \#7: It is nowadays easy to interface augmentation systems of IP with human actors (e.g. producers, suppliers or customers). The integration of this "voice of..." (voice of customers, etc.) is then facilitated through the interface with IP.

\section{$5 \quad$ Possible Use in Real Situations}

To illustrate the way IP can be used, various situations from the APOSAR project are here presented and analyzed, with a 'what-if the IP paradigm was used instead?' view:

i) Aeronautical supply chains involve many partners of very different sizes, exchanging materials with low quantities and high diversity. Within the network, each partner manages his own local priorities for optimizing his service ratio to his immediate customers, even if the decisions made are not the right ones for the interest of the entire supply chain (no global information is locally known).

ii) In large companies, each logistic manager is responsible for a set of suppliers, i.e. is responsible for getting the orders of "his" suppliers on time. In most of the cases, they only know the due date of the orders, but not the slack time introduced by their own production planners. As a consequence, they put a uniform pressure on the suppliers, even if these ones would require the prioritization of the orders.

iii) As a first solution for previous problem, one of the large companies provides information on its inventory level with each order. In this way, the supplier may decide the real urgency of the order if a problem occurs. Nevertheless, the problem remains when several suppliers provide the same part (the decisions made in real time by each supplier are not consolidated).

iv) In order to decrease their prices through time (which is required by their contracts), the suppliers often group orders, but do not always take into account basic information like due dates, with the result of both early and tardy orders.

v) Large companies are often complaining on the lack of visibility of some of their suppliers, due to limited skills on flow management: they would need to know whether their parts have passed some milestones at their supplier's, in order to monitor their fulfillment. This is for instance done by a surface treatment company, who has launched a web site showing the progress of the orders in his workshops. Nevertheless, the effort to maintain this service appears to be too important for being generalized to other suppliers. 
vi) Several cases were mentioned where sub-contractors were waiting for raw materials from their customers, and were warned at the same time by another department that their late in completing the order.

Using the IP paradigm, the global planning of the SC embedded in the IP, periodically updated when synchronization points are met, would address points i) and ii). Constraints can be implemented in the embedded IP information system, which consistence with any new incoming information could be checked: for instance, if the local planning is inconsistent with the global one, an alarm could be triggered (point iv). Checking the consistence of various aspects of the planning (availability of raw materials and due dates for instance, point vi)) could also been made within a given company, before the local information system is embedded in the IP. An IP has intrinsic follow-up capacities. The IP could directly inform the community when it reaches pre-determined milestones, instead of requiring external services in that purpose (point iii)). Point v) is also addressed via the traceability capabilities of the IP.

\section{Conclusion}

In this communication, we have discussed some practical problems linked to information exchange occurring in nowadays supply chains, with a special emphasis on the aeronautical sector. The authors think that the IP paradigm, implying a strong rupture in the way systems are usually considered and designed, could help to address some of these problems and would provide rapid gains by bringing more "natural" solutions to historical problems in SC. IP paradigm is indeed associated to a more "bottom-up" approach, providing emerging behaviour not really anticipated nor designed. Of course, only general considerations, drawn from our knowledge on SC and our past experience in the application of IP in manufacturing, have been presented. Obviously, more detailed research must be led and real implementations miss to prove these intuitions since specific issues may arise. For example, it is clear that designing the structure of the augmentation systems, and specifying their communication with the partners, would require an important effort, including building complex agreements between partners. On physical aspects as well as on logical ones, it is likely that the implementation cost may be important. This is a reason why the case of aeronautical $\mathrm{SC}$ is of specific interest, considering the cost of the parts and the cost of any delay in the delivery of an aircraft. Nevertheless, main obstacles are in our opinion elsewhere. The first one is in the possible nervousness of an emerging or self-organized system based on IP [Barbosa et al., 2012]. Indeed, a SC needs a planned, stable organisation. Getting real time data has always induced the temptation to re-plan in real time, and possibly disorganize the SC (see the influence of the ERP on the planning methods in the 2000's). The second obstacle is more difficult: focal companies of the SC (final assemblers in the case of aeronautical supply chains) have clearly the main interest in the implementation of such system, which can allow them to introduce an eye at their supplier's. This temptation would undoubtedly be a major obstacle for the adoption of the system. On the opposite, such system should be an opportunity to increase mutual trust, which is only possible if a mutual interest is found in the use of the system. 


\section{References}

Arkan, A., Hejazi, S.R.: Coordinating orders in a two echelon supply chain with controllable lead time and ordering cost using the credit period. Comp. \& Ind. Eng. 62, 56-69 (2012)

Barbosa, J., Leitão, P., Adam, E., Trentesaux, D.: Nervousness in dynamic self-organized holonic multi-agent systems. In: Pérez, J.B., Sánchez, M.A., Mathieu, P., Rodríguez, J.M.C., Adam, E., Ortega, A., Moreno, M.N., Navarro, E., Hirsch, B., Lopes-Cardoso, H., Julián, V. (eds.) PAAMS 2012. AISC, vol. 156, pp. 19-28. Springer, Heidelberg (2012)

Ding, H., Guo, B., Liu, Z.: Information sharing and profit allotment based on supply chain cooperation. Int. J. of Prod. Econ. 133(1), 70-79 (2011)

Kiritsis, D.: Closed-loop PLM for intelligent products in the era of the internet of things. Computer-Aided Design 43(5), 479-501 (2011)

Krause, D.R., Handfield, R.B., Tyler, B.B.: The relationship between supplier development, commitment, social capital accumulation and performance improvement. J. of Op. Management 25(2), 528-545 (2007)

McFarlane, D., Giannikas, V., Wong, A., Harrison, M.G.: Intelligent Products in the Supply Chain - 10 Years On. In: IFAC 14th INCOM, pp. 655-660 (2012)

McFarlane, D., Sarma, S., Chirn, J.L., Wong, C.Y., Ashton, K.: The intelligent product in manufacturing control and management. In: 15th IFAC Triennial World Congress, Barcelona, Spain, July 21-26 (2002)

Meyer, G.G., Främling, K., Holmström, J.: Intelligent Products: A survey. Comp. in Ind. 60(3), 137-148 (2009)

Min, H., Zhou, G.H.: Supply Chain Modeling: past, present and future. Comp. \& Ind. Eng. 43, 231-249 (2002)

Ming, Y., Grabot, B., Houe, R.: A taxonomy of the situations of cooperation in supply chains. Comp. \& Ind. Eng. (submitted, 2013)

Nyaga, G.N., Whipple, J.M., Lynch, D.F.: Examining supply chain relationship: Do buyer and supplier perspectives on collaborative relationships differ. J. of Op. Management 28, 101-114 (2010)

Ren, S.J.F., Ngai, E.W.T., Cho, V.: Examining the determinants of outsourcing partnership quality in Chinese small- and medium-sized enterprise. Int. J. of Prod. Res. 48(2), 453-475 (2010)

Sallez, Y., Berger, T., Deneux, D., Trentesaux, D.: The Life Cycle of Active and Intelligent Products: The Augmentation concept. Int. J. of Comp. Int. Manuf. 23(10), 905-924 (2010a)

Sallez, Y., Berger, T., Raileanu, S., Chaabane, S., Trentesaux, D.: Semi-heterarchical control of FMS: From theory to application. Eng. App. Art. Int. 23, 1314-1326 (2010b)

Sarac, A., Absi, N., Dauzère-Pérès, S.: A literature review on the impact of RFID technologies on supply chain management. Int. J. Prod. Econ. 128, 77-95 (2010)

Wadwha, S., Mishra, M., Chan, F.T.S., Ducq, Y.: Effects on information transparency and cooperation on supply chain performance: a simulation study. Int. J. of Prod. Res. 48(1), $145-166$ (2010)

Yang, X., Moore, P., Chong, S.K.: Intelligent products: From lifecycle data acquisition to enabling product-related services. Comp. Comp. in Ind. 60, 184-194 (2009) 